(С) И. Ф. Лозовский, 2015

\title{
Повышение защищённости РЛС обзора от воздействия точечных помех
}

Рассмотрены методы повышения защищённости РЛС обзора от точечных по дальности помех, основанные на селекции сигналов по частоте Доплера, временно́му и угловому положению. Предложено использовать как новые алгоритмы обнаружения сигналов, включающие управляемый частотный порог, цензор импульсов пачки, пораженных несинхронной помехой, так и новые зондирующие сигналы, в том числе пачечные.

Ключевые слова: РЛС обзора, пассивная помеха, «ангел», местный предмет, пачка импульсов, ложная тревога, цель, обнаружение.

\section{Введение}

В настоящей работе под точечной понимается помеха, длительность которой соответствует длительности сигнала, отражённого от точечной по дальности цели. Такие помехи могут образоваться в результате отражений зондирующего сигнала от местных предметов с большой эффективной поверхностью рассеяния (ЭПР), оптически ненаблюдаемых неоднородностей атмосферы, называемых «ангелами», элементов метеообразований или облаков диполей, дающих в условиях турбулентной атмосферы сигнал с аномальными характеристиками, которые существенно отличаются от характеристик в большинстве элементов разрешения. Также точечные помехи могут быть вызваны работой различных радиотехнических средств, случайно или преднамеренно генерирующих импульсные сигналы с параметрами, близкими к параметрам сигналов РЛС обзора (РЛО).

Увеличение интенсивности точечных помех может быть вызвано плохими погодными условиями, сложным рельефом местности, на которой работает радиолокатор, увеличением мощности передатчика РЛО, ответным переизлучением сигналов средствами электронного противодействия и другими факторами. Точечную помеху практически невозможно отличить от сигнала цели, особенно при одноимпульсном зондировании, которое часто используется в РЛО из-за недостатка времени.

Очевидно, что такие помехи могут представлять значительную опасность для РЛО, в первую очередь из-за того, что при их интенсивном воздействии число объектов, обнаруживаемых за один цикл обзора по азимуту, углу места и дальности, может превысить пропускную способность РЛО, составляющую не более 100-200 отметок. Вследствие того, что
РЛО не в состоянии переработать такой объём информации, происходит её информационное подавление и фактически прекращение функционирования.

В существующих РЛО имеются средства подавления точечных помех, включающие [1, 2]:

временную автоматическую регулировку усиления;

межобзорное бланкирование отметок от местных предметов;

череспериодную компенсацию (как двукратную для защиты от местных предметов, так и однократную разностно-временную для борьбы с «ангелами»);

жёсткое ограничение амплитуд и бинарное накопление импульсов пачки;

подавление боковых лепестков диаграммы направленности антенны (ДНА).

Однако, как показывают натурные испытания РЛО и теоретические расчёты, вышеуказанных алгоритмов не всегда бывает достаточно для требуемого подавления помех, а их функционирование может привести к заметному увеличению количества необнаружений сигналов. В связи с этим задача построения эффективной защиты РЛО от точечных помех по-прежнему остается актуальной. В данной работе содержатся основные результаты исследований методов повышения защищённости РЛО от воздействия точечных помех.

Рассматриваемые в статье методы защиты РЛО от точечных помех основаны на селекции сигналов по следующим параметрам:

частоте Доплера, которая у цели обычно выше, чем у точечных пассивных помех;

временно́му положению, которое, в отличие от цели, остаётся постоянным в последующих обзорах для местных предметов или, 
наоборот, изменяется при многократном зондировании в случае несинхронных импульсных помех;

угловому направлению, которое у цели соответствует основному лучу ДНА, в то время как сигналы от точечных местных предметов и импульсные помехи от радиотехнических средств могут поступать и через боковые лепестки ДНА. Сигналы от целей с большой ЭПР, поступающие через боковые лепестки ДНА, также могут считаться точечными помехами.

\section{1. «Ангелы»}

Рассмотрим методы защиты от «ангелов», основанные на селекции сигналов по доплеровской частоте. При наличии жёсткой когерентности от периода к периоду в пачке импульсов в качестве основной альтернативы реализованной в РЛО однократной разностно-временной череспериодной компенсации (ЧПК) используем алгоритм частотного порога с некогерентным накоплением сигналов цели (ЧП-НН) [3]:

$$
\hat{t}=\frac{\frac{1}{N} \sum_{k=1}^{N}\left|x_{k}\right|^{2}}{\frac{1}{N} \sum_{k=1}^{N} \hat{P}_{k}}>C ;
$$$$
\left|\hat{f}_{d}\right|=\frac{1}{2 \pi \cdot \Delta T} \arg \left[\exp \left(j\left(\hat{\theta}\left(T_{2}\right)-\hat{\theta}\left(T_{1}\right)\right)\right)\right]>C_{f} \text {, }
$$

где $x_{k}$ - отсчёты одной дальности в разных периодах повторения когерентной пачки из $N$ импульсов;

$\hat{P}_{k}$ - оценка мощности помех в элементах дальности $k$-го периода, окружающих проверяемый элемент;

$C$ - амплитудный порог;

$C_{f}$ - частотный порог;

$\Delta T$ - вобуляция периода пачки;

$\hat{\theta}\left(T_{k}\right)$ - оценки межпериодных фаз.

Оценки межпериодных фаз $\hat{\theta}\left(T_{k}\right)$ из выражения (2) определяются из соотношения:

$$
\exp \left[j \hat{\theta}\left(T_{k}\right)\right]=\frac{x_{k}^{*} x_{k+1}}{\left|x_{k}^{*} x_{k+1}\right|} .
$$

В неравенствах (1) и (2) сигнал цели считается обнаруженным только в случае одновременного превышения амплитудного (1) и частотного (2) порогов, что и обеспечивает за- щищённость от пассивных помех.

Сравнение характеристик разностновременной ЧПК и алгоритма ЧП-НН проводилось по ряду параметров: число ложных тревог (ЛТ), вероятность правильного обнаружения $P_{d}$ целей разного вида, вероятностноскоростная характеристика (зависимость $P_{d}$ от скорости $V$, сокращённо ВCX). Для расчётов здесь и в дальнейшем использовались модели РЛО сантиметрового диапазона, работающих с длиной волны $\lambda \approx 3-10$ см. Рассматривались следующие виды целей:

«тихоходные», движущиеся со скоростью $V_{\mathrm{c} \min }=200 \mathrm{M} / \mathrm{c}$;

«среднескоростные» $\left(V_{\mathrm{c}}=\left(V_{\mathrm{c} \max }+V_{\mathrm{c} \text { min }}\right) / 2\right)$ $V_{\mathrm{c} \max } \approx 1000 \mathrm{M} / \mathrm{c}$

«разноскоростные», движущиеся с произвольной скоростью в диапазоне $V_{\mathrm{c}}=\left[V_{\mathrm{c} \text { min }}\right.$, $\left.V_{\mathrm{c} \max }\right]$.

Число «ангелов» в зоне обзора $N_{a}$ изменялось случайным образом с равномерным законом распределения до $10^{4}$.

По результатам имитационного моделирования для однократной ЧПК допустимое число ЛТ (не более 100) имело место только при спокойной погоде и относительно небольшом числе помех $\left(N_{a}<10^{3}\right)$. Алгоритм ЧП-НН обеспечил заметное расширение допустимого числа «ангелов» в зоне обзора до единиц тысяч $\left(N_{a}>10^{3}\right)$ и работоспособность РЛО даже в плохих погодных условиях. При этом вероятность правильного обнаружения целей разного вида, включая сравнительно «тихоходные» $V_{\text {c }}=200$ м/с, остаётся довольно высокой.

Для обеспечения заданного числа ЛТ в любых погодных условиях в предложенном алгоритме ЧП-НН приходится устанавливать максимально высокий порог $C_{f}$, что, естественно, приводит к определенному снижению вероятности правильного обнаружения целей с малой радиальной скоростью. Для управления частотным порогом (ЧП) в зависимости от условий приёма в самом простом варианте можно использовать итерационный алгоритм, в котором значение ЧП $C_{f}(k+1)$ для всех элементов разрешения в следующем $(k+1)$-м обзоре зависит от текущего значения ЧП $C_{f}(k)$ и числа отметок в $(k)$-м обзоре $N_{d}(k)$ :

$$
C_{f}(k+1)=C_{f}(k)+\operatorname{sign}\left(N_{d}(k)-N_{\mathrm{o}}\right) \cdot \Delta C_{f} .
$$


где $\Delta C_{f}$ - шаг изменения ЧП;

$N_{\text {o }}$ - номинальное значение числа отметок в одном обзоре;

$\operatorname{sign}($ o) - функция знака.

Для демонстрации эффективности обнаружения целей в РЛО с адаптивным ЧП (АЧП) на рис. 1 показаны ВСХ при разном числе «ангелов» в зоне обзора. Видно, что с увеличением числа «ангелов» расширяется область провала ВСХ в зоне нулевых скоростей для обеспечения требуемого числа ЛТ. В то же время при сравнительно небольшом числе «ангелов» за счёт адаптивного снижения ЧП появляется возможность обнаружения целей с низкой радиальной скоростью $V_{\mathrm{c}}=50-150 \mathrm{M} / \mathrm{c}$.

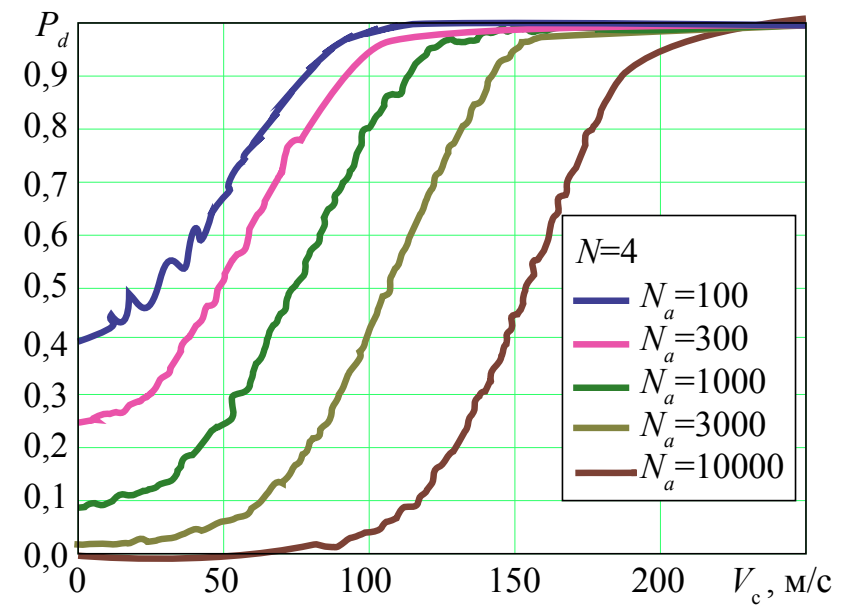

Рис. 1. Вероятностно-скоростная характеристика адаптивного-частотного порога: параметры ангелов $V_{B}=50 \mathrm{м} / \mathrm{c}, \sigma_{f}=40$ Гц, дробно-рациональный спектр

Дальнейшее увеличение подавления «ангелов» возможно за счёт применения в РЛО других типов сигналов, например, пачечных [4], обычно представляющих собой пачку из $N$ $=2 \ldots 8$ импульсов, которые следуют с небольшим (десятки мкс) периодом, обеспечивающим высокие значения межпериодного коэффициента корреляции и потенциально бо́льшее подавление пассивных помех. При этом, однако, должны отсутствовать наложения сигналов от разных областей пассивных помех, что позволяет применять этот метод только в случае наличия точечных или небольшой протяжённости по дальности помех в просматриваемом РЛО угловом направлении.

Для обработки пачечных сигналов предлагается использовать алгоритм ЧП-АКН (АКН адаптивный когерентный накопитель), который выглядит следующим образом:

$$
\left|\mathbf{X}^{T} \mathbf{W}\right|>C
$$

где $\mathbf{X}=\left(x_{1}, x_{2} \ldots, x_{N}\right)^{T}-$ вектор $\mathrm{N}$ принятых сигналов;

$\left.\mathbf{W}=\left(1, \exp \left(-j 2 \pi \hat{f}_{d} T_{i}\right), \ldots \exp \left(-j(N-1) 2 \pi \hat{f}_{d} T_{i}\right)\right)\right)^{T}$

- вектор весовых коэффициентов АКН;

$N$ - количество импульсов в пачке;

$T_{i}$ - период повторения импульсов в пачке;

$C$ - порог обнаружения, зависящий от вероятности ЛТ в условиях шума $P_{f}$.

Оценка частоты Доплера принятого сигнала производится по алгоритму [5]:

$$
\frac{1}{2 \pi T_{i}} \arg \left(\exp \left[j 2 \pi \hat{f}_{d} T_{i}\right]\right)=\frac{\sum_{k=2}^{N} x_{k-1}^{*} x_{k}}{\left|\sum_{k=2}^{N} x_{k-1}^{*} x_{k}\right|} .
$$

Далее по аналогии с (2) используется условие ЧП $-\left|\hat{f}_{d}\right|>C_{f}$. По результатам расчётов величина ЧП в два и более раз меньше, чем в случае разностно-временных оценок, что определяет более высокую видимость «тихоходных» целей и отсутствие существенной необходимости адаптации величины ЧП к помеховой ситуации в зоне обзора РЛО. Важно, что величина ЧП практически не зависит от ширины спектра «ангелов», что определяется высокой межпериодной корреляцией принятых сигналов. Очевидно, такой метод будет сохранять свою работоспособность и в плохих погодных условиях (сильный ветер, грозовые явления и т. п.).

\section{2. Местные предметы}

Методы, аналогичные вышеописанным, можно использовать и для защиты РЛО от точечных местных предметов. В применяемом в настоящее время алгоритме двукратной ЧПК требуемое число ЛТ не обеспечивается при большом количестве точечных местных предметов в зоне обзора, при недостаточной стабильности отражённых от них сигналов. Кроме того, из-за вобуляции периода повторения пачки амплитудно-частотная характеристика имеет провалы, в которых обнаружение цели может отсутствовать. При использовании алгоритма ЧП-НН достигается снижение числа ЛТ до величины, соответствующей пропускной способности РЛО, а ВСХ алгоритма не имеет провалов, что исключает пропуски целей с 
«неблагоприятными» скоростями (рис. 2). При использовании пачечных сигналов для защиты от точечных местных предметов величина ЧП по результатам имитационного моделирования не превышает 100 Гц.

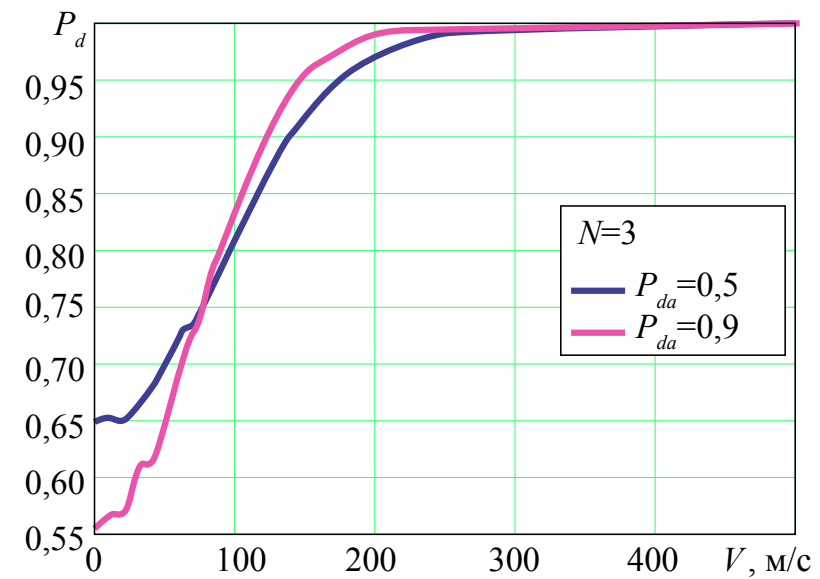

Рис. 2. Вероятностно-скоростная характеристика частотного порога с некогерентным накоплением (точечные местные предметы): $P_{d a}-$ вероятность обнаружения цели в амплитудном режиме

\section{3. Пассивные помехи малой протяжённости}

Следующий вид точечных пассивных помех, с которым приходится встречаться на практике, представляет собой отражения от «блестящих» точек протяжённых пассивных помех, аномальные спектральные характеристики которых в условиях турбулентной атмосферы существенно отличаются от характеристик в большинстве элементов разрешения. Такие отражения плохо подавляются в адаптивных режекторных фильтрах (АРФ) и вызывают существенное увеличение числа ЛТ.

На примере обработки пачечных сигналов рассмотрим следующий алгоритм обнаружения. Принятый после излучения $N$ импульсов вектор сигналов обрабатывается в АРФ $\left(N_{w}+1\right)$-го порядка $\left(N_{w}<N\right)$, достаточном для эффективного подавления конечно-протяжённой пассивной помехи:

$$
y_{i}=\mathbf{X}\left(i: i+N_{w}-1\right) \cdot \mathbf{W}_{r}, i=\overline{1, N-N_{w}+1} .
$$

Полученный из величин $y_{i}$ вектор $\mathbf{Y}=\left(y_{1}, y_{2} \ldots, y_{N-N_{w}+1}\right)^{T}-$ это вектор сигналов на выходе АРФ с весовыми коэффициентами режекторного фильтра $\mathbf{W}_{r}$. Вектор $\mathbf{Y}$ далее подается на АКН, который работает по алгоритму:

$$
z=\mathbf{Y}^{T} \mathbf{W}_{c},
$$

где

$\mathbf{W}_{c}=\left(1, \exp \left(-j 2 \pi \hat{f}_{d} T_{i}\right), \ldots \exp \left(-j\left(N-N_{w}\right) 2 \pi \hat{f}_{d} T_{i}\right)\right)^{T}$
- вектор весовых коэффициентов АКН.

Оценка частоты Доплера каждой компоненты весового вектора $\mathbf{W}_{c}$ производится по алгоритму:

$$
\frac{1}{2 \pi T_{i}} \arg \left(\exp \left[j 2 \pi \hat{f}_{d} T_{i}\right]\right)=\frac{\sum_{k=2}^{N-N_{w-1}+1} y_{k-1}^{*} y_{k}}{\left|\sum_{k=2}^{N-N_{w}+1} y_{k-1}^{*} y_{k}\right|} .
$$

Алгоритм обнаружения однократного пачечного сигнала имеет вид:

$$
|z|>C,\left|\hat{f}_{d}\right|>C_{f}
$$

Для уменьшения числа ЛТ от аномальных элементов конечно-протяжённых пассивных помех в (10) производится бланкирование сигналов с низкой доплеровской частотой на выходе АРФ. В расчётах отклонения спектральных характеристик отражений в «блестящих» точках от характеристик в остальных элементах разрешения брались случайными в диапазоне $g_{p}=0 . .20$ дБ по мощности и $\left|\Delta f_{d}\right| \leq 10^{3}$ Гц по доплеровской частоте. Величина ЧП оказалась близка к требуемому в условиях «ангелов», что обеспечивает достаточную стабильность числа ЛТ и обнаружение целей, движущихся с малыми скоростями.

\section{4. Пассивные помехи большой протяжён- ности}

В условиях пассивных помех большой протяжённости по дальности требуемое число импульсов в пачке с вобуляцией периода составляет не менее 8-16. В связи с этим целесообразно рассмотреть упрощённые алгоритмы обнаружения сигналов, не требующие таких временны́х затрат и снижения темпа обзора РЛО. Такой алгоритм обработки сигналов в зоне протяжённых пассивных помех, в частности, может быть основан на применении вместо адаптивной двукратной разностно-временной ЧПК, способ построения которой приведен в [1]:

$y=x_{1} \exp j \hat{\theta}_{12} \exp (-j \Delta \hat{\theta})-2 x_{2}+x_{3} \exp \left(-j \hat{\theta}_{12}\right),(11)$ где $\Delta \hat{\theta}=\hat{\theta}_{23}-\hat{\theta}_{12}-$ оценка разностной фазы помехи;

$\hat{\theta}_{k k+1}$ - оценки разностных фаз между сигналами от $k$-го и $(k+1)$-го импульсов пачки.

В табл. 1 приведены полученные на имитационной модели данные по параметрам двукратных адаптивной ЧПК (АЧПК2) и 
Таблица 1

Параметры адаптивной и разностно-временной ЧПК

\begin{tabular}{|c|c|c|c|c|c|c|}
\hline \multirow{2}{*}{$\Delta f$, Гц } & \multicolumn{2}{|c|}{$K_{\mathrm{p}}$, дБ } & \multicolumn{2}{c|}{$K_{\mathrm{mB}}$, дБ } & \multicolumn{2}{c|}{$N_{f}$} \\
\cline { 2 - 7 } & АЧПК2 & РВ ЧПК2 & АЧПК2 & РВ ЧПК2 & АЧПК2 & РВ ЧПК2 \\
\hline 20 & 36,6 & 35,3 & 24,9 & 21,8 & 460 & 38 \\
\hline 40 & 30,0 & 28,3 & 18,4 & 15,9 & 329 & 42 \\
\hline 70 & 23,5 & 21,0 & 11,9 & 9,5 & 205 & 43 \\
\hline
\end{tabular}

разностно-временной ЧПК (РВ ЧПК2): коэффициентам подавления $K_{\mathrm{p}}$, подпомеховой видимости $K_{\text {пв }}$ и числу ЛТ $N_{f}$. Спектр пассивных помех был дробно-рациональным шириной $\Delta f=20 \ldots 70$ Гц, мощность помех составляла 40 дБ, число «блестящих» точек в зоне обзора РЛО $N_{t}=10^{3}$. По результатам расчётов алгоритм разностно-временной ЧПК2 уступает адаптивной ЧПК2 до 2,5 дБ в величине коэффициента подавления и до 3 дБ в величине коэффициента подпомеховой видимости. В то же время существенно, в 5-10 раз, снижается число ЛТ от «блестящих» точек пассивных помех.

Снизить мощность остатков пассивных помех на выходе системы обработки и, соответственно, число ЛТ от элементов дальности с аномальными параметрами, можно, в том числе, и с помощью адаптивного аттенюатора сигналов на входе приёмника и «карты помех» [6]. Алгоритм обработки сигналов выражается следующим образом:

$$
\mathbf{X}_{a}=a \mathbf{X}_{p}+\mathbf{X}_{n}, a=\frac{U_{k}}{\sqrt{P}}, y=\mathbf{X}_{a}^{T} \mathbf{W},|y|>C \sqrt{\hat{P}_{y}},
$$

где $\mathbf{X}_{p}=\left(x_{p 1}, x_{p 2} \ldots, x_{p N}\right)^{T}-$ сигналы пассивной помехи в $N$ периодах повторения пачки импульсов;

$\mathbf{X}_{n}=\left(x_{n 1}, x_{n 2} \ldots, x_{n N}\right)^{T}$ - выборочные значения собственного шума приёмника в $N$ периодах повторения пачки импульсов;

$a$ - коэффициент передачи СВЧ аттенюатоpa, устанавливаемый так, чтобы уровень сигналов на входе системы обработки не превышал $U_{k}$ и записываемый для каждого из участков дальности в память («карту помех»);

$P$ - мощность пассивной помехи относительно шума на входе приёмника;

$\mathbf{W}=\left(w_{1}, w_{2}, \ldots w_{N}\right)^{T}-$ весовые коэффициенты ЧПК;

$\hat{P}_{y}$ - оценка мощности остатков помех и шума на выходе ЧПК;

$C-$ порог обнаружения.
В существующих РЛО $U_{k}$ обычно выбирается таким образом, чтобы обеспечивалась линейность обработки сигналов. При $U_{k} \approx \sqrt{P / P_{y}}$ мощность остатков пассивных помех на выходе ЧПК близка к мощности шума приёмника, следовательно, контроль мощности остатков на выходе ЧПК может обеспечить снижение уровня ЛТ от конечно-протяжённых по дальности и точечных пассивных помех. На рис. 3 показаны зависимости числа ЛТ в зоне обзора $N_{f}$ от уровня сигналов, поддерживаемых «картой помех» $U_{k}$ (в зоне обзора имитировалось воздействие $10^{3}$ точечных пассивных помех). В зависимости от параметров спектра пассивных помех число ЛТ снижается до $2-4$-х раз (на рис. 3 до 2-х раз). При использовании такого метода имеет место определённое (до 6 дБ) снижение коэффициента подпомеховой видимости целей $K_{\text {пв }}$.

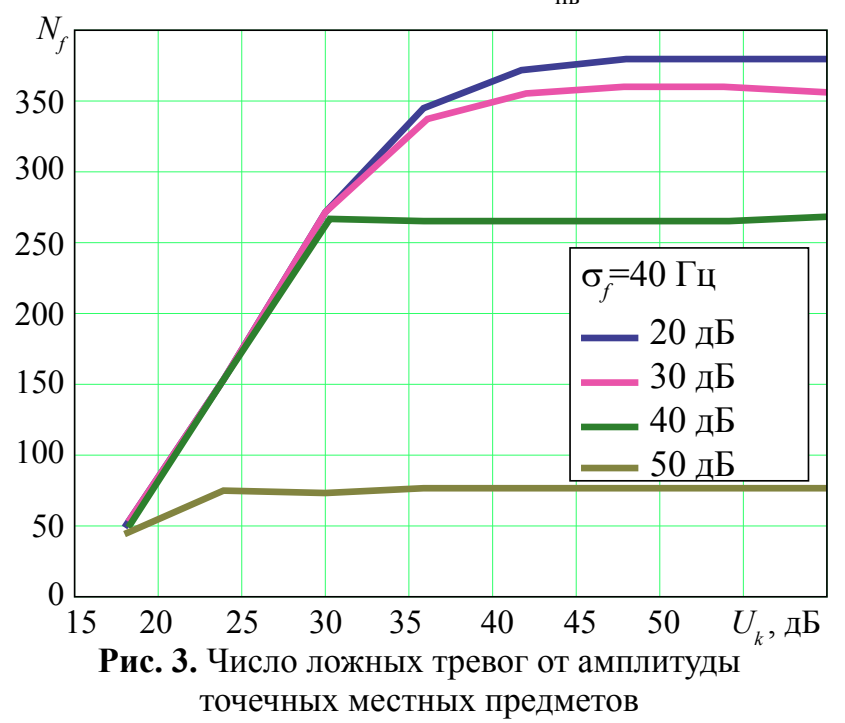

\section{5. Селекция сигналов}

\section{по временному положению}

Другой метод уменьшения числа ЛТ от точечных помех, как уже ранее говорилось, заключается в селекции сигналов по их временному положению. В частности, сигналы, отражённые от местных предметов, в разных обзорах 
имеют одно и то же временное положение, в отличие от сигналов целей, для которых оно будет существенно разным. Реализованный в существующих РЛО метод заключается в бланкировании сигналов, обнаружение которых повторяется от обзора к обзору. При использовании предложенного автором алгоритма с нормировкой дополнительное условие в алгоритме обнаружения имеет вид [6]:

$$
\frac{\left|y_{i}\right|}{\sqrt{\frac{1}{M} \sum_{m=1}^{M}\left|y_{i-m}\right|^{2}}}>C_{n},
$$

где $y_{i}$ - комплексная огибающая сигнала в $i$-м обзоре;

$$
C_{n}=\sqrt{M\left(P_{f}^{-\frac{1}{M}}-1\right)}-\text { порог нормировки; }
$$

$P_{f n}$ - вероятность ложной тревоги при обнаружении точечного местного предмета;

$M$ - число обзоров, в которых в каждом элементе дальности оценивается мощность местных предметов $P_{t}$.

Полученные в данном случае зависимости $P_{f}$ от $P_{t}$ показаны для $M=64$ на рис. 4. При увеличении мощности «местника» величина вероятности ЛТ плавно меняется от номинального уровня в условиях шума $\left(P_{f}=10^{-6}\right) \mathrm{\kappa}$ уровню $P_{f n}$, заданному для условий точечных местных предметов. По результатам имитационного моделирования предложенный алгоритм с нормировкой при его применении в РЛО обеспечивает практически полное подавление отражений от точечных местных предметов.

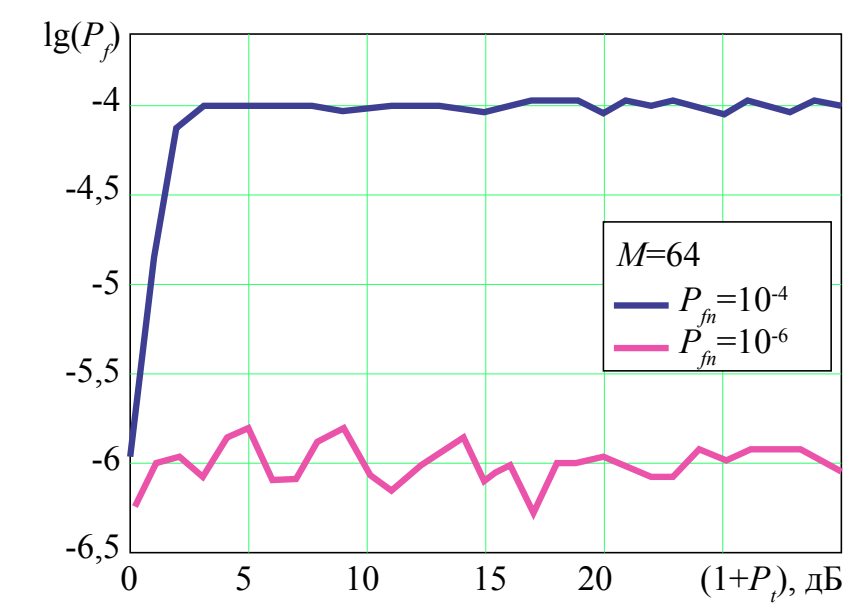

Рис. 4. Зависимости вероятности ложных тревог от мощности остатков точечных местных предметов

\section{6. Точечные несинхронные импульсные помехи}

Селекция сигналов по их временному положению может быть использована и для защиты от точечных несинхронных импульсных помех (ТНИП), излучаемых одновременно работающими радиотехническими средствами или специально с целью подавления РЛО. По сравнению с бинарным накопителем, имеющим потери 1-2дБ, более эффективным способом исключения влияния точечных помех является их цензурирование из выборки накапливаемых отсчётов $x_{1}, x_{2}, \ldots x_{n}$ [7]. Алгоритм цензурирования выглядит следующим образом:

$$
\begin{gathered}
t_{k}=\frac{x_{k}}{\hat{P}_{k}}, \\
k=1, \ldots, n ; \\
t^{(r)}>d \cdot t^{\left(n-N_{t}\right)}, \\
r=n, \ldots, n-N_{t}+1,
\end{gathered}
$$

где $t_{k}$ - нормированные к оценкам мощности помех квадраты амплитуд сигналов;

$d$ - коэффициент взвешивания элемента $t^{\left(n-N_{t}\right)}$, не содержащего точечную помеху, при проверке $N_{t}$ максимальных элементов $t^{(r)}$ на наличие точечных помех;

$N_{t}$ - максимальное число точечных помех в пачке;

$t^{(r)}-r$-я порядковая статистика в выборке из нормированных к оценкам мощности помех отсчётов;

$\hat{P}_{k}$ - оценка мощности помех в элементах дальности $k$-го периода, окружающих проверяемый элемент.

Коэффициент $d$ определяется на имитационной модели таким образом, чтобы потери обнаружения сигнала на фоне шума были минимальны ( 0,1 дБ). Алгоритм накопления в таком случае выглядит следующим образом:

$$
\frac{1}{(n-l)} \sum_{k=1}^{n-l} t^{(k)}>C_{n-l}
$$

где $l$ - число обнаруженных точечных помех (меняется от 0 до $N_{t}$ );

$C_{n-l}-$ порог обнаружения, устанавливаемый таким образом, чтобы при накоплении пачки из $n-l$ импульсов обеспечивался заданный уровень ЛТ.

В табл. 2 приведены данные по числу ЛТ 
Таблица 2

Число ЛТ за обзор

\begin{tabular}{|l|c|c|c|c|}
\hline \multirow{2}{*}{ Вид Тнип } & \multirow{2}{*}{$P_{i}($ дБ $)$} & \multirow{2}{*}{$T_{i}($ мкс $)$} & \multicolumn{2}{c|}{$N_{f}$} \\
\cline { 4 - 5 } & & & АН & АНЦ \\
\hline Разной мощности со случайным периодом & $0 \ldots 50$ & $500 \ldots 4000$ & 187,258 & 0,002 \\
\hline Мощные со случайным периодом & $20 \ldots 50$ & $500 \ldots 4000$ & 205,087 & 0,003 \\
\hline Слабые со случайным периодом & $0 \ldots 20$ & $500 \ldots 4000$ & 74,123 & 0,006 \\
\hline Разной мощности редкие & $0 \ldots 50$ & 4000 & 59,535 & 0,004 \\
\hline Разной мощности частые & $0 \ldots 50$ & 100 & 844,93 & 776,748 \\
\hline Сверхрефракция & $0 \ldots 40$ & 1000 & 76,113 & 0,072 \\
\hline
\end{tabular}

за обзор при использовании алгоритмов амплитудного накопления (AН) и амплитудного накопления с цензором (АНЦ). Определялась эффективность применения амплитудного накопителя с цензором в РЛО. При этом моделировалась ситуация с воздействием на РЛО точечных помех от $N_{i}=100$ источников, мощность помех относительно шума $P_{i}$ менялась в диапазоне от 0 до 50 дБ, период $T_{i}$ составлял от 100 до 4000 мкс при периоде повторения импульсов пачки $T=1000$ мкс. Число импульсов пачки $N=4$, параметры АНЦ: $d=8$ дБ, $N_{t}=1$, $P_{f}=10^{-6}$. Очевидно, при использовании предложенного алгоритма АНЦ обеспечивается допустимое число ЛТ, в отличие от обычного АН.

\section{7. Точечные несинхронные импульсные} помехи и протяжённые пассивные помехи При попадании точечных несинхронных импульсных помех в зону протяжённых пассивных помех задача селекции сигналов по их временному положению существенно усложняется из-за маскирующего действия протяжённой пассивной помехи. Оптимальный адаптивный алгоритм обнаружения когерентной пачки сигналов на фоне пассивных помех имеет вид:

$$
H_{s}=\frac{\left|\mathbf{X}^{T} \mathbf{R}^{-1} \mathbf{S}^{*}\right|^{2}}{\mathbf{S}^{T} \mathbf{R}^{-1} \mathbf{S}^{*}}>C,
$$

где $\mathbf{X}=\left(x_{1}, x_{2}, \ldots, x_{N}\right)^{T}$ - вектор отсчётов сигналов, принятых после излучения $N$ импульсов пачки;

$\hat{\mathbf{R}}^{-1}$ - матрица, обратная к оценочной корреляционной матрице пассивных помех;

$\mathbf{S}=\left(s_{1}, s_{2}, \ldots, s_{N}\right)^{T}$ - вектор ожидаемого сигнала цели.

В условиях точечных помех, поражаю- щих один из периодов пачки, критерий обнаружения сигнала имеет несколько более сложный вид:

$H_{s}=\frac{\left|\mathbf{X}^{T} \hat{\mathbf{R}}^{-1} \mathbf{S}^{*}\right|^{2}}{\mathbf{S}^{T} \hat{\mathbf{R}}^{-1} \mathbf{S}^{*}}>C, d \cdot H_{s}>\max _{\left(I_{i}=1, N\right)}\left(H_{i}\left(I_{i}\right)\right)$,

где $d$ - весовой множитель;

$H_{i}\left(I_{i}\right)$ - решающая статистика обнаружения ТНИП в зондировании с априори неизвестным номером $I_{i}$.

Решающая статистика $H_{i}\left(I_{i}\right)$ из алгоритма (16) определяется из соотношения:

$$
H_{s}=\frac{\left|\mathbf{X}^{T} \hat{\mathbf{R}}^{-1} \mathbf{U}\left(I_{i}\right)\right|^{2}}{\mathbf{U}^{T}\left(I_{i}\right) \hat{\mathbf{R}}^{-1} \mathbf{U}\left(I_{i}\right)},
$$

где $\mathbf{U}\left(I_{i}\right)$ - вектор ТНИП единичной мощности, попавшей в один из импульсов пачки.

Для данного алгоритма были получены зависимости вероятности ЛТ $P_{f, i}$ от мощности точечных помех $P_{i}$ для разных $N, I_{i}$ (рис. 5). За счёт обнаружения и бланкирования точечных помех обеспечивается существенное уменьшение вероятности ЛТ по крайней мере до $\leq 10^{-1} \ldots 10^{-2}$.

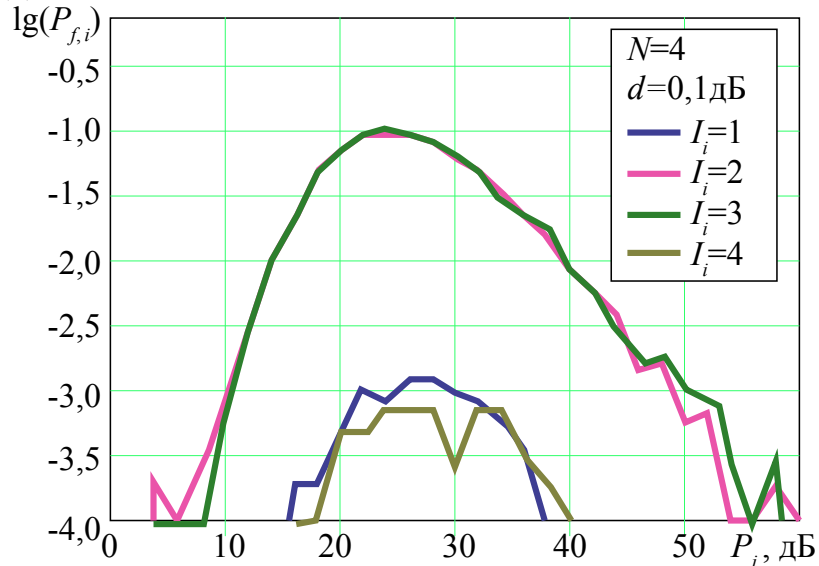

Рис. 5. Зависимости вероятности ложных тревог от мощности точечных некогеретных импульсных помех 


\section{8. Селекция сигнала}

\section{по угловому направлению}

Последним из рассматриваемых является метод селекции сигналов по угловому направлению. Данный метод должен быть эффективен для подавления точечных помех от местных предметов и импульсных помех искусственного происхождения, поступающих в приёмный канал РЛО через боковые лепестки ДНА. Для наиболее общей ситуации обнаружения $N$-импульсной когерентной пачки сигналов на фоне смеси пассивных помех и ложного сигнала (ЛС), принятого по боковым лепесткам ДНА, алгоритм имеет выражение [8]:

$$
\begin{aligned}
& \left|z_{\mathrm{o}}\right|=\left|\mathbf{X}_{\mathrm{o}}^{T} \mathbf{W}_{\mathrm{o}}\right|_{H_{0}}^{\stackrel{H_{1}}{<}} C_{\mathrm{o}} \sqrt{\hat{\sigma}_{\mathrm{o}, w}^{2}} ; \\
& \left|z_{\text {д }}\right|=\left|\mathbf{X}_{\text {д }}^{T} \mathbf{W}_{\text {д }}\right|_{H_{H_{0}}}^{\stackrel{H_{1}}{<}} C_{\text {д }} \sqrt{\hat{\sigma}_{\text {д }, w}^{2}} ; \\
& \left|z_{\mathrm{o}}\right|_{\substack{H_{0} \\
H_{1}}}^{<} C_{\mathrm{o} m} \sqrt{\hat{\sigma}_{\mathrm{o}, w}^{2}}+\left|\hat{\mathbf{A}}_{m}^{T} \cdot \mathbf{W}_{\mathrm{o}}\right| ; \\
& \left|z_{\text {д }}\right|>C_{\text {д }} \sqrt{\hat{\sigma}_{\text {д }, w}^{2}},
\end{aligned}
$$

где $\mathbf{X}_{\mathrm{o}}, \mathbf{X}_{\text {д }}$ - векторы из $N$ комплексных отсчётов в основном и дополнительном каналах (ОК и ДК) соответственно;

$\mathbf{W}_{\text {о }}, \mathbf{W}_{\text {д }}-$ векторы весовых коэффициентов режекторного фильтра (РФ), используемых для подавления пассивных помех в ОК и ДК соответственно;

$\hat{\sigma}_{\text {о, }}^{2}, \hat{\sigma}_{\text {д, }}^{2}-$ соответственно, оценки мощности шума и протяжённых по дальности пассивных помех на выходах РФ в ОК и ДК;

$\hat{\mathbf{A}}_{m}=\lambda^{-1} \mathbf{X}_{d}-$ оценка вектора амплитуд ЛС в OK;

$C_{\text {o }}$ - порог обнаружения в основном канале приёма;

$C_{\text {д }}-$ порог обнаружения в дополнительном канале приёма;

$C_{\text {оm }}-$ разностный порог обнаружения в основном канале приёма;

$\lambda$ - отношение главного лепестка ДНА дополнительного канала к боковым лепесткам ДНА основного канала.

На рис. 6 для одноимпульсного режима показаны зависимости вероятности ЛТ $P_{f}$ от амплитуды точечных местных предметов в ОК

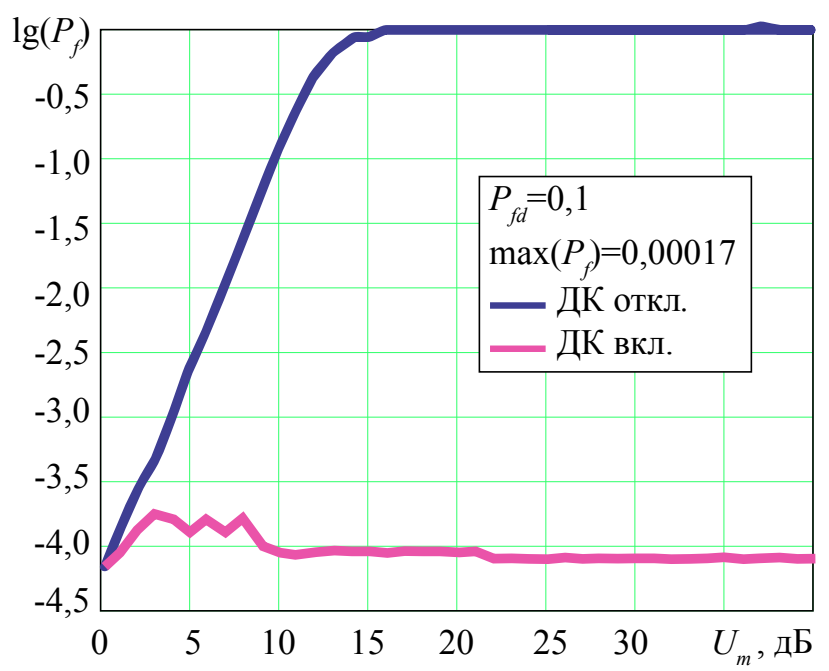

Рис. 6. Зависимости вероятности ложных тревог от амплитуды точечных местных предметов

$U_{m}$ при отключенном и включенном ДК. Рисунок иллюстрирует очевидные преимущества систем с ДК в условиях точечных местных предметов, принятых по боковым лепесткам ДНА. Пороговая мощность обнаруживаемого на фоне шума сигнала за счёт выбора соответствующей величины порога $C_{d}$ увеличилась незначительно ( на 012 дБ).

Проводились расчёты числа ЛТ в РЛО с системой подавления боковых лепестков ДНА (ПБЛ) и без неё в условиях точечных местных предметов. Точечные местные предметы равномерно распределялись по азимуту, их общее число составляло $10^{4}$. Для одноимпульсного режима полученное число ЛТ составило $N_{f}=$ 0,1-0,2 за один цикл обзора по азимуту, углу места и дальности при включённом и $\sim 6 \cdot 10^{5}$ при отключённом ДК. Вероятность обнаружения порогового сигнала, соответствующего вероятности обнаружения 0,5 на фоне шума, снизилась незначительно $-P_{d} \approx 0,47$. В режимах с излучением когерентной пачки из 3-4-х импульсов число ЛТ составило: при включённой ПБЛ - $0-0,65$, при отключенной - до $\sim 7 \cdot 10^{4}$ за обзор. Вероятность обнаружения порогового сигнала составила $0,37-0,51$. В целом включение ПБЛ ДНА в условиях точечных местных предметов приводит к радикальному снижению числа ЛТ, не более одной отметки за обзор, при относительно небольшом уменьшении вероятности обнаружения сигналов целей. Для точечных импульсных помех искусствен- 
ного происхождения получены аналогичные результаты.

\section{Выводы}

1. Алгоритм обнаружения с управляемым по числу отметок в зоне обзора порогом по частоте Доплера является эффективным средством защиты от «ангелов» и точечных местных предметов. Использование зондирующих пачечных сигналов позволит дополнительно повысить защищённость РЛО от данных видов помех. Для существенного снижения числа ложных тревог от «блестящих» точек протяжённых пассивных помех предложены как оптимальные, включающие частотный порог на выходе АРФ, так и упрощенные алгоритмы.

2. Предложенный алгоритм с нормировкой обеспечивает практически полное подавление отражений от точечных местных предметов в РЛО. Полученные оптимальный и упрощенный алгоритмы обнаружения сигналов на фоне смеси пассивных и несинхронных импульсных помех обеспечивают заметное снижение числа ложных тревог.

3. Предложенный алгоритм цензурирования периодов, пораженных импульсной помехой, при обнаружении некогерентной пачки импульсов, обеспечивает в РЛО существенное снижение числа ЛТ.

4. Алгоритм селекции сигналов по угловым направлениям применён для защиты от точечных помех, принятых по боковым лепесткам ДНА, и при моделировании показал свою высокую эффективность.

\section{Список литературы}

1. Лозовский И. Ф. Построение и эффективность разностно-временных компенсаторов точечных движущихся помех // Радиолокация,

навигация, связь : XI Международная конференция. Воронеж: ОАО «Концерн «Созвездие», 2005. T. 3. C. 1386-1397.

2. Кузьмин С. 3. Основы теории цифровой обработки радиолокационной информации. М.: Сов. радио, 1974. 432 с.

3. Лозовский И. Ф. Эффективность частотного порога при разностно-временном подавлении точечных движущихся помех // Радиолокация, навигация, связь : XI Международная конференция. Воронеж: ОАО «Концерн «Созвездие», 2005. T. 3. С. 1398-1405.

4. Лозовский И. Ф. Эффективность обработки пачечных сигналов в условиях локально протяжённых и точечных пассивных помех // Вопросы радиоэлектроники. Сер. Общие вопросы радиоэлектроники. 2006. Вып. 1. С. 38-47. 5. Попов Д. И. Синтез измерителей доплеровской фазы сигнала // Радиотехника. 1991. № 2. C. $36-39$.

6. Лозовский И. Ф. Построение и характеристики систем обнаружения с ПУЛТ в условиях помех от местных предметов // Современные проблемы создания и эксплуатации радиотехнических систем : тр. 4-й науч.-практ. конф. Ульяновск: УлГТУ, 2004. С. 34-37.

7. Лозовский И. Ф. Алгоритмы защиты от несинхронных сигналов при обнаружении некогерентной пачки импульсов // Материалы VII Международной конференции АПЭП-2004. Новосибирск: НГТУ, 2004. Т. 4. С. 33-40.

8. Лозовский И. Ф. Построение и моделирование радиолокационных обнаружителей сигналов с дополнительным некогерентным каналом приёма // Радиотехника. 2006. № 12. С. 4-9

\section{Поступила 26.02.14}

Лозовский Игорь Филиппович - доктор технических наук, старший научный сотрудник, начальник отдела ОАО «НПО НИИИП-НЗиК», г. Новосибирск.

Область научных интересов: цифровая обработка, обнаружение радиолокационных сигналов. 